\title{
Estudante universitário: fatores que contribuem para o consumo de substâncias psicoativas
}

\author{
University student: Factors that contribute to the consumption of psychoactive substances \\ Estudiante universitario: factores que contribuyen al consumo de sustancias psicoactivas
}

Recebido: 10/02/2021 | Revisado: 14/02/2021 | Aceito: 07/03/2021 | Publicado: 14/03/2021

\author{
Ana Patricia Fonseca Coelho Galvão \\ ORCID: https://orcid.org/0000-0003-3376-5678 \\ Faculdade de Ciências Médicas da Santa Casa de São Paulo, Brasil \\ E-mail: apfcoelho@gmail.com \\ Cintia Maria de Lima Barbosa \\ ORCID: https://orcid.org/0000-0003-0968-5387 \\ Universidade Ceuma, Brasil \\ E-mail: enf.cintiabarbosa@gmail.com \\ Francisca Bruna Arruda Aragão \\ ORCID: https://orcid.org/0000-0002-1191-0988 \\ Universidade de São Paulo, Brasil \\ E-mail: aragao_bruna@usp.br \\ Ricardo Riyoiti Uchida \\ ORCID: https://orcid.org/0000-0002-4209-8830 \\ Faculdade de Ciências Médicas da Santa Casa de São Paulo, Brasil \\ E-mail: rruchida@uol.com.br
}

\begin{abstract}
Resumo
O consumo abusivo de álcool, tabaco, e outras substâncias, se caracterizam como problema de saúde pública mundial. No Brasil, o consumo destas substâncias tem influência da mídia, e a falta de políticas públicas, fiscalização e controle de vendas, permitem que o acesso a estas substâncias e o consumo precoce. Dessa maneira, o objetivo foi descrever os fatores que contribuem para o consumo de substâncias psicoativas entre estudantes universitários. Trata-se de um estudo transversal e descritivo, realizado em uma universidade privada localizada no município de São Luís-MA. Participaram 451 alunos matriculados nos cursos de Enfermagem, Medicina, Odontologia e Psicologia. Utilizaram-se os instrumentos para coleta de dados: Questionário de dados sociodemográficos; Escala para Avaliação de Desordens devido ao uso de Álcool (AUDIT) e Cannabis Abuse Screening Test - CAST. Para a análise dos dados obtidos, foi utilizado o software SPSS (versão 21), para obter as estatísticas descritivas. Dos entrevistados, (80,8\%) eram do sexo feminino e (19,0\%) eram do sexo masculino, com idade média entre 21 e 25 anos (53,7\%), As SPA mais consumidas por esses estudantes foram respectivamente Álcool, maconha e estimulantes. E ao se tratar dos fatores associados, foram encontradas as seguintes associações: religião, o apoio institucional, ter sofrido agressão, envolvimento em agressões após uso de bebida alcoólica, dirigir alcoolizado, ter sido passageiro de um motorista alcoolizado, idade que consumiu bebida alcoólica, e uso de estimulantes.
\end{abstract}

Palavras-chave: Estudante; Consumo de álcool na faculdade; Usuários de drogas.

\begin{abstract}
The abusive consumption of alcohol, tobacco, and other substances is characterized as a worldwide public health problem. In Brazil, the consumption of these substances has media influence, and the lack of public policies, inspection and sales control, allow access to these substances and early consumption. This way, the objective was to describe the factors that contribute to the consumption of psychoactive substances among university students. This is a crosssectional and descriptive study, carried out in a private university located in the municipality of São Luís-MA. There were 451 students enrolled in the Nursing, Medicine, Dentistry, and Psychology courses. The instruments were used for data collection: Sociodemographic Data Questionnaire; Scale for Evaluation of Disorders due to Alcohol Use (AUDIT) and Cannabis Abuse Screening Test - CAST. The SPSS software (version 21) was used to obtain descriptive statistics for the analysis of the data obtained. Of the respondents, (80.8\%) were female and (19.0\%) were male, with an average age between 21 and 25 years (53.7\%). The most consumed SPA by these students were respectively Alcohol, Marijuana and Stimulants. The following associations were found when dealing with the associated factors: religion, institutional support, having suffered aggression, involvement in aggression after the use of alcoholic beverages, drunk driving, having been a passenger of an alcoholic driver, age who consumed alcoholic beverages, and use of stimulants. Keywords: Student; Consume of alcohol in college; Drug users.
\end{abstract}




\begin{abstract}
Resumen
El consumo abusivo de alcohol, tabaco y otras sustancias se caracteriza por ser un problema de salud pública en todo el mundo. En Brasil, el consumo de estas sustancias está influenciado por los medios de comunicación, y la falta de políticas públicas, supervisión y control de las ventas permiten el acceso a estas sustancias y el consumo temprano. Así, el objetivo fue describir los factores que contribuyen al consumo de sustancias psicoactivas entre los estudiantes universitarios. Se trata de un estudio transversal y descriptivo, realizado en una universidad privada ubicada en el municipio de São Luís-MA. Participaron 451 estudiantes matriculados en los cursos de Enfermería, Medicina, Odontología y Psicología. Se utilizaron los siguientes instrumentos de recogida de datos: un cuestionario de datos sociodemográficos, una Escala de Evaluación de Trastornos por Consumo de Alcohol (AUDIT) y un Test de Detección del Abuso de Cannabis - CAST. Para el análisis de los datos obtenidos, se utilizó el programa informático SPSS (versión 21) para obtener estadísticas descriptivas. De los encuestados, $(80,8 \%)$ eran mujeres y $(19,0 \%)$ eran hombres, con una edad media entre 21 y 25 años $(53,7 \%)$, Las SPA más consumidas por estos estudiantes fueron respectivamente Alcohol, marihuana y estimulantes. Y en cuanto a los factores asociados, se encontraron las siguientes asociaciones: religión, apoyo institucional, haber sufrido agresiones, participación en agresiones después de beber alcohol, conducir bajo los efectos del alcohol, haber sido pasajero de un conductor ebrio, edad a la que se consumió el alcohol y uso de estimulantes.
\end{abstract}

Palabras clave: Estudiante; Consumo de alcohol en la universidad; Usuarios de drogas.

\title{
1. Introdução
}

O uso de substâncias psicoativas (SPA's) não tem início recente, percebemos que seu consumo se difunde como uma prática milenar, e somente há seis décadas seu uso tem sido enfrentado como um problema (Ferraz et al., 2018). Sobretudo, atuam no cérebro, alterando o psiquismo e, consequentemente, afetando o comportamento humano. Gonçalves et al. (2019) acrescenta que o consumo abusivo de álcool, tabaco, e outras substâncias, se caracterizam como problema de saúde pública mundial.

Nessa perspectiva, a United Nations Office on Drugs and Crimes-UNODC (2019), destacou que em 2017 cerca de 271 milhões pessoas entre 15-64 anos fizeram uso de alguma substância lícita e/ou ilícita, este número representa 5\% da população mundial, o estudo revela ainda que globalmente cerca de 53 milhões de pessoas sofrem de algum transtorno decorrente ao uso de álcool e drogas.

Destacamos que no Brasil, o consumo destas substâncias tem influência da mídia, e a falta de políticas públicas, fiscalização e controle de vendas, permite que o acesso a estas substâncias seja fácil a adolescentes, permitindo que contato comece precocemente, merecendo destaque todos os aspectos de vulnerabilidade (De Araújo et al., 2019).

Acreditamos que por esse motivo houve do aumento do consumo de substâncias psicoativas nessa população, além de estar relacionado aos problemas cotidianos, como os de cunho universitário e sociais, onde, para encaixar nesse o estudante deve se adequar as condições estabelecidas pelo meio, que na maioria das vezes está relacionada ao consumo excessivo de álcool e drogas (Silva, 2017).

O álcool assume um papel de droga mais bem aceita na sociedade, e tendo assim seu consumo descriminado. Além disso, o seu baixo custo se torna um propiciador para um consumo maior, e podendo assim se tornar um gatilho para o a inserção do consumo de drogas ilícitas (Kawano, 2019).

Esse fácil acesso, pode ocasionar danos á saúde, com também outros problemas, como acidentes automobilísticos, violência sexual, violência física, dentre outro, desta maneira o álcool como problema de saúde publica é de alta relevância, por afetar não somente quem faz seu uso, como também as pessoas ao redor deste individuo (Ramalho, 2019).

Dentre todas as substancias ilícitas a maconha, cientificamente, canabis sativa, ganha destaque, por ser a mais consumida, e ser considerada "leve" pelos estudantes em comparação a outras drogas, tem seu consumo beneficiado e facilitado em relação a outras, podendo se tornar a porta de entrada para experimentar as substâncias psicoativa (SPA's) mais nocivas como o crack, e cocaína. (Vieira \& Santos, 2020).

Entretanto, existem outras substâncias psicoativas que podem ser alvo de consumo entre universitários. Podemos incluir a cocaína, e as sintetizadas em laboratórios como as anfetaminas, estimulantes do SNC como ritalina, além dos esteroides 
anabolizantes (Ferro et al., 2019).

Os estudantes universitários devem merecer um enfoque diferenciado em relação ao uso de todas as substâncias ilícitas, pois, futuramente, são eles que levarão as noções básicas de saúde à comunidade. Desta forma, se torna essencial conhecer o padrão de consumo, as atitudes e o conhecimento em relação às drogas entre esses alunos com objetivo de descrever os fatores que contribuem para o consumo de substâncias psicoativas entre estudantes universitários.

\section{Metodologia}

Trata-se de um estudo transversal e descritivo com direcionalidade temporal prospectiva. A pesquisa foi realizada na Universidade Ceuma em 2 Campi - Anil e Renascença, a Uni-CEUMA, uma instituição privada fundada há 27 anos no Estado do Maranhão e a partir de 2012 se insere no trinômio ensino, pesquisa e extensão.

A população se constituiu por 451 estudantes dos cursos de graduação de Enfermagem, Odontologia, Psicologia e Medicina. Foram incluídos estudantes universitários do primeiro ao último ano, maiores de 18 anos devidamente matriculados. E excluídos os que estavam de atestado médico, licença maternidade, não matriculados e menores de 18 anos.

Foram utilizados os seguintes instrumentos para coleta de dados: Questionário de dados sociodemográficos; Escala para Avaliação de Desordens devido ao uso de Álcool (AUDIT) e Cannabis Abuse Screening Test - CAST.

Destacamos que, a Escala para Avaliação de Desordens devido ao uso de Álcool (AUDIT), foi criada pela Organização Mundial da Saúde com o objetivo de rastrear consumo excessivo de álcool e consiste em 10 questões sobre o consumo de álcool. Cada resposta recebeu uma pontuação de 0 a 4 e, então, a pontuação final varia de 0 a 40 , sendo que, quanto mais alta, maior a probabilidade de o consumo estar afetando a saúde e segurança do indivíduo. A correção da AUDIT categoriza os indivíduos em quatro zonas: Zona I (baixo risco); Zona II (com risco); Zona III (uso nocivo); Zona IV (possível dependência). A consistência interna no instrumento na amostra do presente estudo foi satisfatória $(\alpha=0,87)$ (Saunders et al., 1993).

E Cannabis Abuse Screening Test - CAST, um questionário pelo Observatório Francês das Drogas e Toxico de pendências contém seis questões que visam identificar tendência a abuso de maconha, a partir de seis padrões de uso da droga, nos últimos seis meses: uso antes do meio-dia, uso sozinho, ocorrência de problemas de memória devido ao uso, ter recebido conselho de familiares ou amigos a reduzir o uso, tentativa sem sucesso de diminuir ou cessar o uso e ocorrência de problemas, tal como rixas, discussões, acidentes e maus resultados na escola devido ao uso do produto. A pontuação foi depois classificada em quatro níveis de dependência: 0 - sem risco, 1 a 2 - risco baixo, 3 - risco moderado e 4 a 6 - risco elevado. A consistência interna da escala nessa amostra foi satisfatória $(\alpha=0,94)$ (Legleye et al., 2015).

A coleta de dados seguiu todas as recomendações em razão dos casos de infecção por COVID-19. Primeiramente, se entrou em contato com os coordenadores dos cursos de graduação. Posteriormente, agendou-se com os líderes de turma do primeiro ao último ano, explicando a pesquisa, apresentando seus objetivos, com intuito de agendar as coletas de dados, que foi realizada de forma online através da plataforma Google Forms. E o acessá-lo, o participante deparou-se com o Termo de Consentimento Livre e Esclarecido (TCLE), que ressalta o caráter voluntário e sigiloso da pesquisa. Somente após concordar com o termo, o aluno poderá responder o questionário.

Para a análise dos dados obtidos, foi utilizado o software SPSS (versão 21), com o intuito de obter as estatísticas descritivas (média, desvio padrão e frequências), correlação Pearson e testes de associação Qui-Quadrado de Pearson.

Esta pesquisa está ligada a um projeto maior, intitulado, "SAÚDE MENTAL DO ESTUDANTE UNIVERSITÁRIO: Fatores protetores e fatores de vulnerabilidade", que obedeceu ao posicionamento ético, norteou-se a partir das recomendações éticas dispostas nas Normas e Diretrizes que regulamentam a pesquisa envolvendo seres humanos, estabelecidas na Resolução 466/12 do Conselho Nacional de Saúde, sendo aprovada no Comitê de Ética em Pesquisa através do Parecer Consubstanciado $\mathrm{n}^{\circ}$ 4.127.772. 


\section{Resultados e Discussão}

Os dados da pesquisa serão apresentados em forma de tabelas e discutidos conforme sua distribuição para melhor visualização e compreensão.

Tabela 1 - Caracterização dos dados sociodemográficos. São Luis, 2020.

\begin{tabular}{|c|c|c|}
\hline Variáveis & $\mathrm{N}$ & $\%$ \\
\hline \multicolumn{3}{|l|}{ Sexo } \\
\hline Masculino & 88 & 19,5 \\
\hline Feminino & 362 & 80,3 \\
\hline Ausente & 1 & 0,2 \\
\hline \multicolumn{3}{|l|}{ Idades } \\
\hline Entre 18 e 20 anos & 140 & 31,0 \\
\hline Entre 21 e 25 anos & 242 & 53,7 \\
\hline Entre 26 e 30 anos & 40 & 8,9 \\
\hline Acima de 31 de anos & 29 & 6,4 \\
\hline \multicolumn{3}{|l|}{ Estado Civil } \\
\hline Solteiro(a) & 419 & 92,9 \\
\hline Casado(a) & 28 & 6,2 \\
\hline Divorciado(a) & 3 & 0,7 \\
\hline Separado(a) & 1 & 0,2 \\
\hline \multicolumn{3}{|l|}{ Filhos } \\
\hline Não tenho filhos & 410 & 90,9 \\
\hline Sim, um filho & 16 & 3,5 \\
\hline Sim, dois filhos & 16 & 3,5 \\
\hline Sim, três ou mais & 6 & 1,3 \\
\hline Ausente & 3 & 0,6 \\
\hline \multicolumn{3}{|l|}{ Mora com quem } \\
\hline Pais & 210 & 46,6 \\
\hline Familiares & 152 & 33,7 \\
\hline Amigos & 28 & 6,2 \\
\hline Sozinho(a) & 55 & 12,2 \\
\hline Ausente & 6 & 1,3 \\
\hline \multicolumn{3}{|l|}{ Religião } \\
\hline Católica & 194 & 43,0 \\
\hline Evangélica & 160 & 35,5 \\
\hline Espírita & 15 & 3,3 \\
\hline Ateu & 12 & 2,7 \\
\hline Outra & 68 & 15,1 \\
\hline Ausente & 2 & 0,4 \\
\hline
\end{tabular}

Fonte: Autores (2020).

A caracterização sociodemográfica mais detalhada da amostra apresentada na tabela 1, sendo composta 451 estudantes com idades entre 18 e 64 anos $(M=22,92$; DP = 5,68), a maioria entre 21 e 25 anos $(53,7 \%)$, do sexo feminino $(80,3 \%)$ e solteiro(a) (92,9\%). Grande parte dos participantes relatou não ter filhos (90,9\%), residir com pais (46,6\%) ou familiares (33,7\%), e ter religião católica $(43,0 \%)$ ou evangélica $(35,5 \%)$. 
Tabela 2 - Caracterização Agressão e uso de drogas, estimulantes, relação sexual sem proteção e anabolizantes. São Luis, 2020.

\begin{tabular}{|c|c|c|}
\hline Variável & $\mathrm{N}$ & $\%$ \\
\hline \multicolumn{3}{|l|}{ Agressão no último ano } \\
\hline Não & 399 & 88,5 \\
\hline $\operatorname{Sim}$ & 49 & 10,9 \\
\hline Ausente & 3 & 0,6 \\
\hline \multicolumn{3}{|c|}{ Se sim, consumiu bebidas alcoólicas? } \\
\hline Não & 130 & 28,8 \\
\hline Sim & 11 & 2,4 \\
\hline Ausente & 310 & 68,7 \\
\hline \multicolumn{3}{|l|}{ Você já dirigiu alcoolizado? } \\
\hline Não & 368 & 81,6 \\
\hline $\operatorname{Sim}$ & 77 & 17,1 \\
\hline Ausente & 6 & 1,3 \\
\hline \multicolumn{3}{|c|}{ Você já esteve no carro com um motorista alcoolizado? } \\
\hline Não & 151 & 33,5 \\
\hline Sim & 297 & 65,9 \\
\hline Ausente & 3 & 0,6 \\
\hline \multicolumn{3}{|c|}{ Se você consumiu bebidas alcoólicas, com quantos anos? } \\
\hline Menor de 12 anos & 10 & 2,2 \\
\hline Entre 12 e 15 anos & 86 & 19,0 \\
\hline Entre 16 e 18 anos & 155 & 34,4 \\
\hline Maior de 18 anos & 54 & 12,0 \\
\hline Nunca consumiu/Ausente & 146 & 32,4 \\
\hline \multicolumn{3}{|c|}{ Se você consumiu maconha, com quantos anos? } \\
\hline Menor/igual a 15 anos & 11 & 2,4 \\
\hline Entre 16 e 18 anos & 28 & 6,2 \\
\hline Maior de 18 anos & 43 & 9,5 \\
\hline Nunca consumiu/Ausente & 369 & 81,9 \\
\hline \multicolumn{3}{|c|}{$\begin{array}{l}\text { Você já teve relação sexual desprotegida (sem preservativo) com } \\
\text { parceiro casual? }\end{array}$} \\
\hline Não & 233 & 51,7 \\
\hline Sim & 205 & 45,5 \\
\hline Ausente & 13 & 2,8 \\
\hline
\end{tabular}

Antes de entrar na faculdade, você já havia feito uso de algum dos seguintes estimulantes, com a intenção de melhorar o rendimento dos estudos? Selecione todos os que já tiver usado.

Ritalina

112,4

Outros

Opitimemory

Nunca usou/Ausente

Se você respondeu à pergunta anterior, como conseguiu a medicação?

Amigos

$15 \quad 3,3$

Prescrição médica feita em uma consulta formal e adequada

$11 \quad 2,4$

Comprando sem receita

Receita médica sem consulta adequada

Ausente

Se respondeu à pergunta anterior, você acredita que melhorou?

$\begin{array}{lcc}\text { Não } & 17 & 3,8 \\ \text { Sim } & 18 & 4,0 \\ \text { Ausente } & 416 & 92,2 \\ \text { Você já fez uso de anabolizantes? } & 410 & 90,9 \\ \text { Não } & 8 & 1,8 \\ \text { Sim } & 33 & 7,3 \\ \text { Ausente }\end{array}$

Fonte: Autores (2020). 
As SPA's que apresentaram maior relevância em relação ao seu consumo no presente estudo, foram respectivamente, álcool, maconha e estimulantes. Não havendo resultados significativos para o uso de anabolizantes, e outras substâncias.

Esses achados se assemelham com os achados de Pelicioli et al. (2017), que trás o álcool como substância mais consumida pela população em estudo, e Vincenzi et al. (2017) que relata a maconha como segunda substância mais consumida entre os universitários.

Tabela 3 - Distribuição dos níveis de risco para uso de álcool e maconha. São Luis, 2020.

\begin{tabular}{lcc}
\hline & N & $\%$ \\
AUDIT (N =403) & 290 & 72,0 \\
Baixo Risco & 90 & 22,3 \\
Com Risco & 6 & 1,5 \\
Uso Nocivo & 17 & 4,2 \\
Dependência & & \\
CAST (N = 327) & 308 & 94,2 \\
Sem Risco & 9 & 2,8 \\
Risco Baixo & 6 & 1,8 \\
Risco Moderado & 4 & 1,2 \\
Risco Elevado & & \\
\hline
\end{tabular}

Fonte: Autores (2020).

Na Tabela 3, os estudantes apresentaram médias baixas nos escores das escalas AUDIT e CAST. A partir das classificações das escalas foi possível encontrar diferentes níveis de risco entre os estudantes.

Em relação ao álcool, a maioria dos estudantes foi classificada com baixo risco para uso abusivo de álcool (72,0\%), seguido de algum risco $(22,3 \%)$, dependência $(4,2 \%)$ e uso nocivo $(1,5 \%)$.

A representatividade dos dados referidos pode ser comparada com o estudo de Marinho et al. (2019), onde 53\% estudantes dos estudantes entrevistados apresentaram consumo de baixo risco, $34 \%$ consumo de risco, $7 \%$ uso nocivo ou consumo de alto risco, enquanto, $6 \%$ apresentaram provável dependência do álcool.

Partindo para a variável do uso de maconha, grande parte $(94,2 \%)$ foi classificada sem risco, 2,8\% com risco baixo, 1,8\% com risco moderado e 1,2\% com risco elevado. A concordância dos dados é baseada no estudo de Pereira et al. (2020), realizado com acadêmicos das áreas das ciências humanas, exatas e biológicas, entre o primeiro e o ultimo ano de graduação, no qual 86,7\% não apresentaram nenhum risco, 20,5\% apresentaram risco moderado, e 5,4 apresentam risco alto.

Tabela 4 - Representação do Teste Qui-Quadrado de Pearson entre as classificações de uso de álcool, maconha e variáveis sociodemográficas, atividade física e apoio institucional e de colegas. São Luis, 2020.

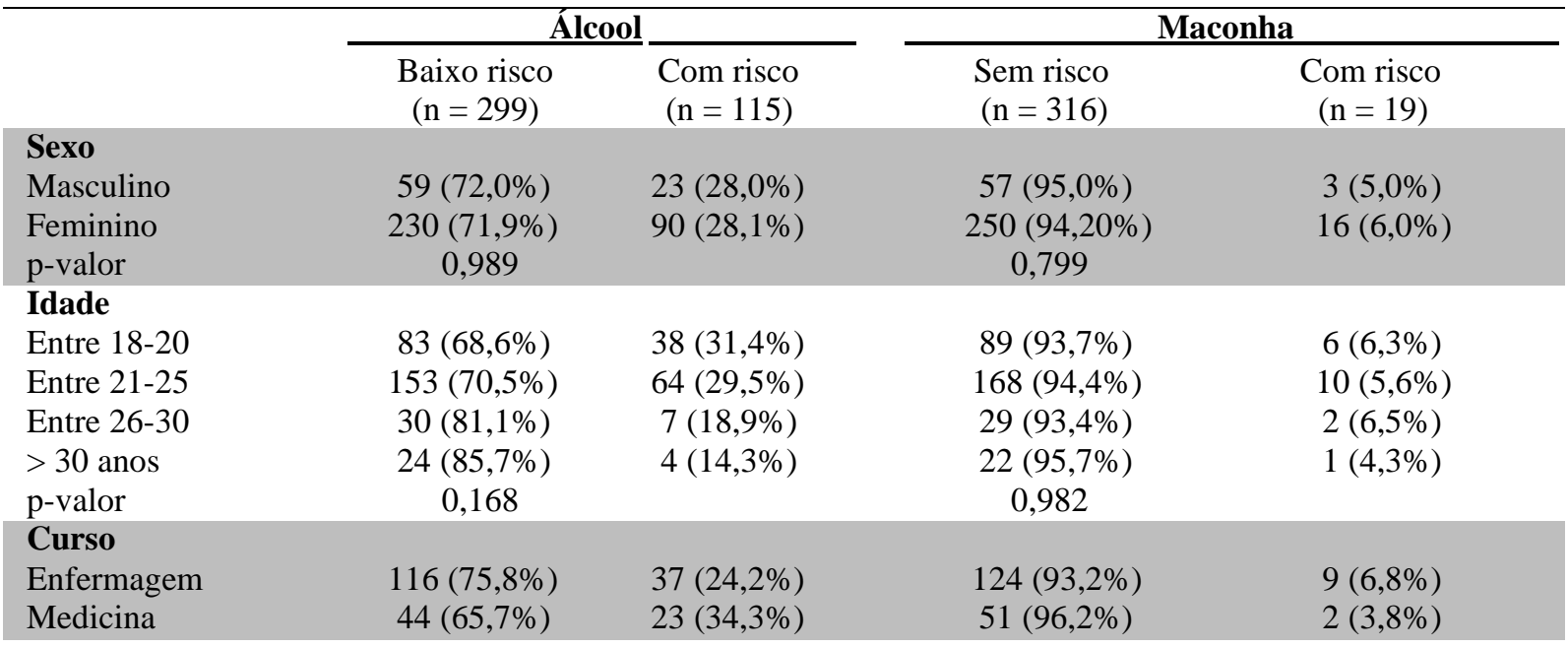




\begin{tabular}{|c|c|c|c|c|}
\hline $\begin{array}{l}\text { Odontologia } \\
\text { Psicologia } \\
\text { p-valor }\end{array}$ & $\begin{array}{c}36(62,1 \%) \\
94(75,8 \%) \\
0,103\end{array}$ & $\begin{array}{l}22(37,9 \%) \\
30(24,2 \%)\end{array}$ & $\begin{array}{c}43(100 \%) \\
89(91,8 \%) \\
0,229\end{array}$ & $\begin{array}{l}0(0,0 \%) \\
8(8,2 \%)\end{array}$ \\
\hline $\begin{array}{l}\text { Religião } \\
\text { Católica } \\
\text { Evangélica } \\
\text { Espírita } \\
\text { Ateísmo } \\
\text { Outras } \\
\text { p-valor }\end{array}$ & $\begin{array}{c}118(65,6 \%) \\
112(85,5 \%) \\
7(53,8 \%) \\
4(33,3 \%) \\
47(72,3 \%) \\
<0,001\end{array}$ & $\begin{array}{c}62(34,4 \%) \\
19(14,5 \%) \\
6(46,2 \%) \\
8(66,7 \%) \\
18(27,7 \%)\end{array}$ & $\begin{array}{c}134(96,4 \%) \\
101(93,5 \%) \\
12(92,3 \%) \\
8(72,7 \%) \\
51(94,4 \%) \\
0,031\end{array}$ & $\begin{array}{l}5(3,6 \%) \\
7(6,5 \%) \\
1(7,7 \%) \\
3(27,3 \%) \\
3(5,6 \%)\end{array}$ \\
\hline $\begin{array}{l}\text { Atividade Física } \\
\text { Não pratica } \\
\text { Pratica } \\
\text { p-valor }\end{array}$ & $\begin{array}{c}126(73,3 \%) \\
164(71,0 \%) \\
0,617\end{array}$ & $\begin{array}{l}46(26,7 \%) \\
67(29,0 \%)\end{array}$ & $\begin{array}{c}136(94,4 \%) \\
172(94,0 \%) \\
0,861\end{array}$ & $\begin{array}{c}8(5,6 \%) \\
11(6,0 \%)\end{array}$ \\
\hline $\begin{array}{l}\text { Apoio } \\
\text { Instituição } \\
\text { Não } \\
\text { Sim } \\
\text { p-valor }\end{array}$ & $\begin{array}{c}187(68,5 \%) \\
100(78,7 \%) \\
0,034\end{array}$ & $\begin{array}{l}86(31,5 \%) \\
27(21,3 \%)\end{array}$ & $\begin{array}{c}213(95,5 \%) \\
94(91,3 \%) \\
0,128\end{array}$ & $\begin{array}{c}10(4,5 \%) \\
9(8,7 \%)\end{array}$ \\
\hline $\begin{array}{l}\text { Apoio } \\
\text { Colegas } \\
\text { Não } \\
\text { Sim } \\
\text { p-valor }\end{array}$ & $\begin{array}{c}76(74,5 \%) \\
211(70,8 \%) \\
0,473\end{array}$ & $\begin{array}{l}26(25,5 \%) \\
87(29,2 \%)\end{array}$ & $\begin{array}{c}79(95,2 \%) \\
226(93,8 \%) \\
0,639\end{array}$ & $\begin{array}{c}4(4,8 \%) \\
15(6,2 \%)\end{array}$ \\
\hline $\begin{array}{l}\text { Agressão } \\
\text { Não } \\
\text { Sim } \\
\text { p-valor }\end{array}$ & $\begin{array}{c}267(75,2 \%) \\
21(46,7 \%) \\
<0,001\end{array}$ & $\begin{array}{l}88(24,8 \%) \\
24(53,3 \%)\end{array}$ & $\begin{array}{c}272(94,1 \%) \\
36(94,7 \%) \\
0,878\end{array}$ & $\begin{array}{l}17(5,9 \%) \\
2(5,3 \%)\end{array}$ \\
\hline $\begin{array}{l}\text { Agressão depois } \\
\text { de bebida } \\
\text { Não } \\
\text { Sim } \\
\text { p-valor }\end{array}$ & $\begin{array}{c}77(63,6 \%) \\
2(20,0 \%) \\
0,007\end{array}$ & $\begin{array}{c}44(36,4 \%) \\
8(80,0 \%)\end{array}$ & $\begin{array}{c}89(95,7 \%) \\
9(90,0 \%) \\
0,426\end{array}$ & $\begin{array}{c}4(4,3 \%) \\
1(10,0 \%)\end{array}$ \\
\hline $\begin{array}{l}\text { Dirigiu } \\
\text { Alcoolizado } \\
\text { Não } \\
\text { Sim }\end{array}$ & $\begin{array}{c}249(76,9 \%) \\
36(48,6 \%) \\
<0,001\end{array}$ & $\begin{array}{l}75(23,1 \%) \\
38(51,4 \%)\end{array}$ & $\begin{array}{c}253(94,8 \%) \\
52(91,2 \%) \\
0,303\end{array}$ & $\begin{array}{c}14(5,2 \%) \\
5(8,8 \%)\end{array}$ \\
\hline $\begin{array}{l}\text { Motorista } \\
\text { Alcoolizado } \\
\text { Não } \\
\text { Sim } \\
\text { p-valor }\end{array}$ & $\begin{array}{c}119(94,4 \%) \\
169(61,5 \%) \\
<0,001\end{array}$ & $\begin{array}{c}7(5,6 \%) \\
106(38,5 \%)\end{array}$ & $\begin{array}{c}103(96,3 \%) \\
202(93,1 \%) \\
0,253\end{array}$ & $\begin{array}{c}4(3,7 \%) \\
15(6,9 \%)\end{array}$ \\
\hline $\begin{array}{l}\text { Relação sexual } \\
\text { sem proteção } \\
\text { Não } \\
\text { Sim }\end{array}$ & $\begin{array}{c}165(82,5 \%) \\
115(59,6 \%) \\
<0,001\end{array}$ & $\begin{array}{l}35(17,5 \%) \\
78(40,4 \%)\end{array}$ & $\begin{array}{c}157(95,7 \%) \\
145(92,4 \%) \\
0,200\end{array}$ & $\begin{array}{c}7(4,3 \%) \\
12(7,6 \%)\end{array}$ \\
\hline $\begin{array}{l}\text { Idade álcool } \\
<12 \text { anos } \\
12-15 \text { anos } \\
16-18 \text { anos } \\
>18 \text { anos } \\
\text { p-valor }\end{array}$ & $\begin{array}{c}7(77,8 \%) \\
38(45,8 \%) \\
98(68,5 \%) \\
39(73,6 \%) \\
0,001\end{array}$ & $\begin{array}{c}2(22,2 \%) \\
45(54,2 \%) \\
45(31,5 \%) \\
14(26,4 \%)\end{array}$ & $\begin{array}{c}8(100 \%) \\
57(89,1 \%) \\
112(94,1 \%) \\
34(97,1 \%) \\
<0,001\end{array}$ & $\begin{array}{c}0(0 \%) \\
7(10,9 \%) \\
7(5,9 \%) \\
1(2,9 \%)\end{array}$ \\
\hline $\begin{array}{l}\text { Estimulante } \\
\text { Não } \\
\text { Sim } \\
\text { p-valor }\end{array}$ & $\begin{array}{c}274(74,5 \%) \\
16(45,7 \%) \\
<0,001\end{array}$ & $\begin{array}{l}94(25,5 \%) \\
19(54,3 \%)\end{array}$ & $\begin{array}{c}281(94,3 \%) \\
27(93,1 \%) \\
0,793\end{array}$ & $\begin{array}{c}17(5,7 \%) \\
2(6,9 \%)\end{array}$ \\
\hline $\begin{array}{l}\text { Anabolizante } \\
\text { Não } \\
\text { Sim } \\
\text { p-valor }\end{array}$ & $\begin{array}{c}264(71,4 \%) \\
5(62,5 \%) \\
0,585\end{array}$ & $\begin{array}{c}106(28,6 \%) \\
3(37,5 \%)\end{array}$ & $\begin{array}{c}285(94,4 \%) \\
6(85,7 \%) \\
0,334\end{array}$ & $\begin{array}{l}17(5,6 \%) \\
1(14,3 \%)\end{array}$ \\
\hline
\end{tabular}


Fonte: Autores (2020).

Na Tabela 4, traz a representação do Teste Qui-Quadrado de Pearson entre as classificações de uso de álcool, maconha e variáveis sociodemográficas, atividade física e apoio institucional e de colegas, dentre outros.

Essas associações das classificações de risco, foram dicotomizadas para uso de álcool ( 0 = Baixo risco e $1=$ Com risco $)$ e maconha $(0=$ Sem risco; 1 = Com risco). E posteriormente, associadas com as variáveis coletadas no estudo, a fim de conhecer os principais fatores de risco evidenciados nos estudantes universitários.

Os dados apresentados foram associados significativamente ao risco de álcool a religião (p < 0,001), o apoio institucional $(\mathrm{p}=0,034)$; ter sofrido agressão $(\mathrm{p}<0,001)$, envolvimento em agressões após uso de bebida alcoólica $(\mathrm{p}=$ 0,007), dirigir alcoolizado ( $\mathrm{p}<0,001$ ), ter sido passageiro de um motorista alcoolizado ( $<<0,001$ ), idade que consumiu bebida alcoólica ( $\mathrm{p}=0,001)$, e uso de estimulantes $(\mathrm{p}<0,001)$.

Quanto ao sexo, não houve discrepância em relação aos níveis de risco, tanto para homens (28,0\%), como mulheres $(28,1 \%)$. Contudo, o sexo feminino apresentou maior risco que o masculino, corroborando com outros estudos, como o de Maciel e Vargas (2017), no qual houve uma predominância do sexo feminino, e $(64,52)$ dessas mulheres fazem uso de risco do álcool. Para o autor, uma possível explicação para esse comportamento, é a independência feminina, a mulher vem conquistando um papel de igualdade dentro da sociedade, esse fator pode influenciar na adoção de comportamentos anteriormente tipificados como do sexo masculino.

Em relação à religião, a mesma mostrou-se como fator protetor para evangélicos, já que somente $(14,5 \%)$ dos que se declaram evagelicos, possuem risco. Esse resultado se mostra similar ao trabalho de Rodrigues Júnior et al. (2020), e a maior incidência de consumo entre os espíritas. Contudo, De Araújo et al. (2018) em seu estudo, apresenta uma associação entre os usuários de álcool com indivíduos que se declaravam ateus, corroborando com o observado no presente trabalho.

Algumas pesquisas enfatizam que, a religião possui fator protetor ao se tratar do consumo de drogas, no qual, indivíduos que possuam uma crença ou que se envolvam em algum tipo de atividade religiosa apresentem menor predisposição de se tornar usuário (De Araújo et al., 2018).

Em consonância aos resultados apresentados acima, 51.4\% dos que afirmam, já ter dirigido alcoolizado possuem risco, e ter sido passageiro de um motorista alcoolizado, também mostrou ser um fator associado ao risco do consumo, visto que, $38,5 \%$ daqueles que foram passageiros demostraram possuir risco.

Em um trabalho desenvolvido por Antoniasse Júnior e Gaya (2015), no qual foi constituído por uma amostra de 123 estudantes universitários, e tinha como objetivo verificar os comportamentos de risco relacionados ao uso de álcool e outras drogas, constatou-se que $40 \%$ já dirigiram sobre efeito do álcool, 68,2\% já pegaram carona com motorista também alcoolizado, 16,4\% já haviam se envolvido em acidentes de trânsito sob efeito de álcool. Através deste estudo, pode-se constatar que, os estudantes apresentavam comportamentos de risco relacionando ao consumo de SPA's, como envolvimento em acidentes de trânsito, expondo a vulnerabilidade dessa população, e mostrando a necessidade de politicas para prevenção dos universitários.

Além disso, a tendência do início de consumo precoce pode ser observa da no estudo, visto que 54,2\% afirmam que consumiram álcool pela primeira vez entre 12 e 15 anos. Ratificando esse achado, uma pesquisa realizada por Rodrigues (2020), demostrou 55,9\% dos entrevistados relataram começar o uso antes de completar 18 anos de idade, sendo que 13,7\% iniciaram aos 14 anos ou menos. Observamos uma potente relação entre a precocidade do consumo dessa substancias com o surgimento de problemas relacionado ao seu consumo, e também a probabilidade de posteriormente o surgimento de uma possível dependência.

Em relação a ter sofrido agressão, em uma revisão de literatura realizada por De Carvalho et al., publicada em 2017, os autores ressaltam que ter sido vitima de agressão principalmente durante a infância e adolescência é um fator que pode levar ao consumo de álcool. Este dado corrobora com os achados do presente estudo, onde 53,3\% das pessoas que se envolveram em 
algum tipo de agressão possuem risco para o consumo abusivo de álcool.

No que tange a variável sobre o apoio institucional, 31,5\% não percebem o apoio da instituição tem risco. Em um estudo realizado por Lima et al. (2018) sobre importância da Universidade sobre uso de álcool e de outras drogas, a autora entrevistou acadêmicos de uma universidade em São Paulo, no qual estes, demonstraram se sentir desamparados pela instituição, e apontam uma falta de diálogo por parte da mesma sobre a temática. Sendo importante que, as instituições de ensino superior invistam em formações e ações de extensão como redutores de danos, atuando no cotidiano e nas interações de sociabilidade.

Outro fator identificado no estudo foi o uso de drogas como a Ritalina, Conccerta, Veivance. No presente estudo, que 54,3\% dos estudantes fizeram uso de substâncias e apresentam risco, são pertencentes ao sexo feminino, e desses, 3,3\% afirmam ter conseguido através de amigos, e sem receituário médico. Além do mais, dos 54,3\% que afirmam o consumo, 3,8\% dizem perceber melhora em relação ao rendimento de suas atividades acadêmicas.

Em consonância com esses achados, Cordeiro e Pinto (2017) em suas pesquisas, trás que 9,8\% dos estudantes, já haviam utilizado algum estimulante cerebral, e que esse consumo era realizado sem prescrição médica.

Estudos apontam o aumento da capacidade cognitiva como o principal motivador para o consumo dessas substâncias. Essa hipótese se confirma, pelo fato estar ligado às dificuldades que o ensino superior exige, podendo levar os jovens ao uso de substâncias que lhes permitam conciliar suas atividades acadêmicas com a vida pessoal (Da Graça, 2013)

Em relação ao uso abusivo de maconha, se associou significativamente apenas a religião $(p=0,031)$, visto que 27,3\% dos ateus tem risco; e com a idade que usou álcool ( $<<0,001)$, sendo maior risco entre os que consumiram álcool entre 12 e 15 anos $(10,9 \%)$. A falta de variáveis associadas ao risco no uso da maconha pode ser explicada pelo baixo consumo na amostra estudada.

\section{Conclusão}

Podemos concluir que uma parcela significativa dos estudantes utiliza ou em algum momento utilizou alguma SPA's, apontando que os universitários estão inseridos numa população de vulnerabilidade, e imersos a fatores cotidianos que podem levar ao abuso de substâncias psicoativas.

Nos achados, a droga mais utilizada foi o álcool. Contudo, há consumo significativo de outras substâncias, como a maconha e estimulantes, sendo que grande parte dos usuários dessas substâncias, iniciou o uso anterior ao ingresso à universidade.

As variáveis mais associadas ao consumo foram a religião, o apoio institucional, ter sofrido agressão, envolvimento em agressões após uso de bebida alcoólica, dirigir alcoolizado, ter sido passageiro de um motorista alcoolizado, idade que consumiu bebida alcoólica, e uso de estimulantes. E a caracterização de aspectos psicossociais, da saúde mental e frequência de uso de substâncias psicoativas em uma população vulnerável como a universitária, podem servir de importante fonte para elaboração programas de apoio psicológico, e conscientização dentro do ambiente universitário.

Esperamos que, por meio dessa pesquisa, possamos contribuir para a investigação sobre fatores ligados ao consumo de SPA`s entre estudantes universitários, e, assim, detectar precocemente e prevenir esse tipo de problema.

\section{Referências}

Antoniasse Júnior, G., \& Gaya, C. M. (2015). Implicações do uso de álcool, tabaco e outras drogas na vida do universitário. Rev Bras Promoç Saúde, 1(28):6774. http://dx.doi.org/10.5020/18061230.2015.p67

Cordeiro, N., \& Pinto, R. M. C. (2017). Consumo de estimulantes cerebrais em acadêmicos da área da saúde na cidade de Ponta Grossa-PR. Visão Acadêmica, 18(2):23-45. http://dx.doi.org/10.5380/acd.v18i2.53234

Da Graça, C. S. G. (2013). Consumo de estimulantes cerebrais nos estudantes de Medicina da Universidade da Beira Interior. 2013. 50 f. Dissertação (Mestrado) - Faculdade de Ciências da Saúde, Universidade da Beira Interior, Covilhã, 2013. 
Da Silva, P. D. C. (2017). Investigar o consumo de substância psicoativas entre universitários ingressantes de uma instituição pública. 2017. 54f. Trabalho de Conclusão de Curso (Graduação em Enfermagem) - Universidade Federal de Mato Grosso, Instituto de Ciências da Saúde, Sinop, 2017. https://bdm.ufmt.br/bitstream/1/959/1/TCC-2018-P\%c3\%89RICLES\%20DONAR\%20CUNHA\%20DA\%20SILVA.pdf

De Araújo, J. L., Costa, S. M. C., Monteiro, P. M. F., Rego, A. R. F., \& Do Nascimento, E. G. C. (2019). Consumo de alcool entre universitários do interior do nordeste brasileiro. Revista de atenção à saúde, 17(59):88-94. http://dx.doi.org/ 10.13037/ras.vol17n59.5837 ISSN 2359-4330

De Araujo, C. M., Vieira, C. X., \& Mascarenhas, C. H. M. (2018). Prevalência do consumo de drogas lícitas e ilícitas por estudantes universitários. SMAD, Rev. Eletrônica Saúde Mental Álcool Drog, 14(3):144-50. http://dx.doi.org/10.11606/issn.1806-6976.smad.2018.000342

De Carvalho, A. P., Da Silva, T. C., Valença, P. A. M., Santos, C. F. B. F., Colares, V., \& Menezes, V. A. (2017). Consumo de álcool e violência física entre adolescentes: quem é o preditor? Ciênc. saúde coletiva [online]., 22(12):4013-20. https://doi.org/10.1590/1413-812320172212.06172016.

Ferraz, L., Piato, A. L. S., Anzolin, V., Matter, G. R., \& Busato, M. A. (2018). Substâncias psicoativas: o consumo entre acadêmicos de uma universidade do sul do Brasil. Momento-Diálogos em Educação, 27(1):371-86. https://doi.org/10.14295/momento.v27i1.6850

Ferro, L. R. M., Trigo, A. A., Oliveira, A. J., Almeida, M. A. R., Tagava, R. F., Meneses-Gaya, C., \& Rezende, M. M. (2019). Estresse percebido e o uso de álcool, tabaco e outras drogas entre universitários. Saúde e Pesqui., 12(3): 73-581. http://dx.doi.org/10.17765/2176-9206.2019v12n3p573-581

Gonçalves, J. S., Fava, S. M. C. L., Alves, A. C., \& Dázio, E. M. R. (2019). Reflexões acerca do panorama de consumo de álcoole/ou outras drogas entre estudantes universitários. Revista de Enfermagem do Centro- Oeste Mineiro, 9(1): 1-9, e2594. http://dx.doi.org/10.19175/recom.v9i0.2594

Kawano, A. N. (2019). Consumo de álcool e outras drogas por universitários brasileiros da área da saúde: uma revisão integrativa. Trabalho de conclusão de curso (Graduação em Enfermagem) - Faculdade de Medicina, Universidade Federal de Uberlândia, Uberlândia, 2019. https://repositorio.ufu.br/bitstream/123456789/28304/1/Consumo\%c3\%81lcoolOutras.pdf

Legleye, S., Guignard, R., Richard, J. B., Kraus, L., Pabst, A., \& Beck, F. (2015). Propriedades do Teste de Triagem do Abuso de Cannabis (CAST) na população em geral. Int J Methods Psychiatr Res., 24(2):170-83. http://dx.doi.org/10.1002/mpr.1465

Lima, K. H. M., Da Silva, C. G., \& Mendes, R. (2018). Drogas e álcool na universidade: proibições, silenciamentos e diálogos. Temas em Educação e Saúde, 14(1):156-72. https://doi.org/10.26673/rtes.v14.n1.2018.11302

Maciel, M. E. D., Vargas, D. (2017). Consumo de álcool entre estudantes de enfermagem. Rev Fund Care Online, 9(1):64-70. http://dx.doi.org/10.9789/21755361.2017.v9i1.64-70

Marinho, V. L., Da Silva, J. B. F., Teixeira, K. S., Dias, A. R., \& Rosário, R. R. (2019). Consumo de álcool entre estudantes de uma universidade pública da região sul do Tocantins. Revista Cereus, 11(4):2-10, 2019. 10.18605/2175-7275/cereus.v11n4p2-10

Pelicioli, M., Barelli, C., Gonçalves, C. B. C., Hahn, S. R., \& Scherer, J. I. (2017). Perfil do consumo de álcool e prática do beber pesado episódico entre universitários brasileiros da área da saúde. Jornal Brasileiro de Psiquiatria, 66(3):150-6. http://dx.doi.org/ 10.1590/0047-2085000000164

Pereira, A. R., Pontual, A. A. D., Santos, J. L. F., Corradi-Webster, C. M. (2020). Uso de drogas entre universitários de uma universidade federal de Minas Gerais. Revista Valore, 5(1):1-15, e-5023. https://doi.org/10.22408/reva502020434e-5023

Ramalho, A. A. (2019). O consumo de álcool e outras drogas entre alunos ingressantes em cursos da área de saúde. 2019. 36f. Trabalho de Conclusão de Curso (Graduação em Enfermagem) - Faculdade de Ciências da Educação e Saúde, Centro Universitário de Brasília, Brasília, 2019. https://repositorio.uniceub.br/jspui/bitstream/prefix/13622/1/21506006.pdf

Rodrigues Júnior, G. A., Queiroz, F. A., Lima, H. N., Nóbrega, L. S., \& Oliveira, I. R. N. (2020). Fatores associados ao uso de substâncias psicoativas em estudantes de uma universidade pública do sul do Maranhão. Rev Med (São Paulo), 99(3):220-9. http://dx.doi.org/10.11606/issn.1679-9836.v99i3p220-229

Rodrigues, A. L. M., Leão, J. A., \& Morais, L. S. S. (2020). Uso de álcool, tabaco e outras substâncias psicoativas entre discentes do curso de Medicina: um estudo transversal. Para Res Med J., 4(1):1-16,e40. http://10.4322/prmj.2019.040

Saunders, J. B., Aasland, O. G., Babor, T. F., Grant, M. \& Fuente, J. R. (1993). Development of the Alcohol Use Disorders Identification Test (AUDIT : WHO Collaborative Project on Early Detection of Persons with Harmful Alcohol Consumption-II / J. B. Saunders ... [et al.]. Addiction 1993, 88(6):791-804. https://apps.who.int/iris/handle/10665/51167

United Nations Office on Drugs and Crimes-UNODC (2019). Relatório Mundial sobre Drogas, UNODC launches World Report on Drugs, Viena, 26 de junho de 2019. <https://www.unodc.org/lpo-brazil/pt/frontpage/2019/06/relatrio-mundial-sobre-drogas-2019_-35-milhes-de- pessoas-em-todo-o-mundo-sofrem-detranstornos-por-uso-de-drogas--enquanto- apenas-1-em-cada-7-pessoas-recebe-tratamento.html.

Vieira, A. B. \& Santos, E. V. L. (2020). Uso de drogas ilícitas por acadêmicos do curso de medicina: uma revisão bibliométrica. Revista temas em saúde, 20(1):451-474. http://dx.doi.org/10.29327/213319.20.1-26

Vincenzi, T., Mario, D. N., Cericato, G. O., Portilio, M. N., \& Rigo, L. (2017). Maconha como a segunda substância psicoativa mais comumente usada entre estudantes. J Hum Growth Dev., 27(2):244-52. http://dx.doi.org/10.7322/jhgd.125827 